\title{
Proteinuria Thresholds Are Irrational: A Call for Proteinuria Indexing
}

\author{
Timothy J. Ellam Meguid El Nahas
}

The Sheffield Kidney Institute, Sheffield, UK

\section{Key Words}

Albuminuria - Proteinuria - Glomerular filtration rate

\begin{abstract}
Current guidelines for chronic kidney disease (CKD) diagnosis, referral and management are based on absolute thresholds of proteinuria/albuminuria with no reference to the residual nephron mass or function. This is illogical since the severity of proteinuria is a direct reflection of the number of filtering nephrons as well as their pathology and the capacity of the tubules to reabsorb filtered protein/albumin. The current simplistic approach to proteinuria may also compromise its usefulness as a robust guide to appropriate treatment, e.g. preferential use of inhibitors of the renin-angiotensin-aldosterone system. The routine measurement of the urinary protein/albumin:creatinine ratio (PCR/ACR) and estimated glomerular filtration rate (eGFR) gives rise to the opportunity to index proteinuria for renal function (i.e. a PCR:eGFR or ACR:eGFR ratio). Since both PCR/ACR and eGFR are reflections of quantities assessed per unit body surface area, this is a logical approach to the assessment of proteinuria/albuminuria. We advocate a consideration of the benefits of indexing PCR/ACR for eGFR to optimise treatment decisions based on proteinuria/albuminuria.
\end{abstract}

\section{KARGER}

Fax +41613061234 E-Mail karger@karger.ch www.karger.com

\section{Introduction}

With the perceived increase in number of people suffering from chronic kidney disease (CKD), nephrologists and primary care physicians are increasingly relying on proteinuria/albuminuria for the detection, classification and management of CKD. In this respect, nephrologists are fortunate in having at their disposal such a readily measurable biomarker with profound diagnostic, prognostic and therapeutic implications. Proteinuria measurement is an integral component of national and international guidelines for appropriate referral and treatment of patients with CKD $[1,2]$. Also, threshold proteinuria levels have been used to identify patients most likely to derive particular benefit from inhibitors of the renin-angiotensin-aldosterone system (RAAS) and to define optimal blood pressure targets. Currently advocated proteinuria thresholds, however, make no adjustment for the functioning nephron mass through which the protein leak is occurring. Consequently, nephrologists may be failing to make optimal use of this biomarker and are neglecting the reasoning behind its significance. Consider for example 2 patients with a urinary albumin:creatinine ratio (ACR) of $1,000 \mathrm{mg} / \mathrm{g}$ but with glomerular filtration rates (GFR) of 100 and $10 \mathrm{ml} / \mathrm{min} / 1.73 \mathrm{~m}^{2}$, respectively. It is clear that for each patient the protein leak across an in-

Dr. Tim Ellam, MD, MRCP

Sheffield Kidney Institute - Sorby Wing, Northern General Hospital

Sheffield S5 7AU (UK)

Tel. +44114305 2010, Fax +441142266446

E-Mail T.Ellam@sheffield.ac.uk 


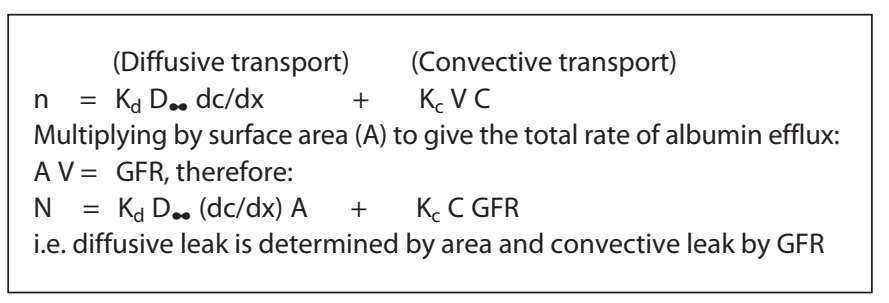

Fig. 1. Determinants of glomerular albumin leak, considering the glomerulus as a semipermeable membrane. $\mathrm{n}=$ Albumin movement at a point in the membrane; $\mathrm{D}_{\infty}=$ albumin diffusivity (constant at $\left.37^{\circ}\right) ; \mathrm{dc} / \mathrm{dx}=$ albumin concentration gradient; $\mathrm{V}=$ fluid flow velocity; $\mathrm{C}=$ local albumin concentration; $\mathrm{N}=$ total albumin efflux across the membrane; $\mathrm{A}=$ total glomerular surface area available; GFR = total GFR; $\mathrm{K}_{\mathrm{d}}, \mathrm{K}_{\mathrm{c}}=$ constants for the membrane determining rate of albumin flux by diffusion and convection, respectively. Modified by the pathology increasing permeability to albumin and therefore the measures of interest.

dividual glomerulus and nephron will be very different. Current guidelines would not, however, distinguish between them on the basis of proteinuria. Similarly, current guidelines for CKD classification defining CKD stages 1 and 2 as people suffering from microalbuminuria do not take into consideration whether the individual with an ACR of $30 \mathrm{mg} / \mathrm{g}$ has a GFR of $120 \mathrm{ml} / \mathrm{min} / 1.73 \mathrm{~m}^{2}$ or one of $60 \mathrm{ml} / \mathrm{min} / 1.73 \mathrm{~m}^{2}$. They would both be labelled as suffering from CKD according to the current KDOQI classification but may have very different prognostic implications.

\section{Determinants of Glomerular Protein Leak}

With regard to glomerular disease, proteinuria quantification is informative in that it reflects an underlying pathology causing a change in the permeability properties of the glomerular filtration barrier. To compare the pathologies of different patients, those factors that influence total protein leak independently of filtration barrier permeability should therefore be adjusted for. This also applies to the assessment of changes in the filtration barrier permeability of an individual over time or following interventions. Factors determining protein (albumin) leak are shown in figure 1. Protein traffic across a leaking glomerular filtration barrier in disease states may be the result of diffusive and/or convective transport, though the relative contributions of each of these are unclear [3]. As shown in figure 1, if diffusion is the dominant mechanism of protein leak, then total glomerular surface area would theoretically be the desirable denominator for proteinuria correction. If convection predominantly drives protein leak, then correcting for GFR would be optimal.

In practice, since the glomerular surface area is unknown, correcting proteinuria for some measure of GFR is the only clinically available option. GFR itself of course provides a reflection of available glomerular surface area and capillary pressure so is a useful correction factor whether diffusion or convection predominates. The (theoretical) difference between correcting for GFR and correcting for glomerular surface area will depend on the extent to which GFR per unit of glomerular surface area varies. This is unknown since although changes in single nephron filtration rate may occur in disease states, these changes may be driven by the adaptive increased glomerular capillary pressure/perfusion that accompanies glomerulomegaly and/or increased diffusive surface area. In the setting of filtration equilibrium, whereby equalization of oncotic and hydrostatic pressures results in ultrafiltration ceasing before the end of the glomerular capillary, diffusion of protein may continue independently of filtration. Thus the variations in single glomerular convective and diffusive capacities may clearly be confounders complicating any simplifying model.

Tubular handling of filtered proteins also affects the ultimate leak of protein into the urine [4]. The extent to which such a mechanism determines the overall extent of proteinuria is not quantifiable clinically. However, even if the view is taken that tubular dysfunction is the main determinant of urinary protein leak as suggested by some [5] or in instances of tubulointerstitial disease, the same argument for GFR indexing applies: assessment of the degree of tubular pathology causing reabsorption failure and proteinuria should require correction for the filtered protein load per nephron. Tubular proteinuria corrected for filtered load of protein will then reflect the fraction of filtered protein that each tubule fails to reabsorb - hence reflecting the tubular pathology.

The exposure of proximal tubules to heavy proteinuria will also vary depending on the number of filtering nephrons: for an equal amount of proteinuria, let us say 1,000 $\mathrm{mg} / \mathrm{g}$, the exposure of proximal tubules will vary depending on whether the GFR is 100 or $10 \mathrm{ml} / \mathrm{min}$. Such a difference in GFR will reflect a hugely different number of nephrons with considerable variation in the filtered load of protein per nephron and consequent potential tubular exposure and toxicity.

Indexing for GFR is thus a logical argument when applied to either albuminuria or proteinuria. For the purposes of fractional excretion measurement, it is theoreti- 
Fig. 2. Fractional excretion of albumin relative to creatinine (A) and relative to eGFR (B). Alb urine$_{\text {, }} \mathrm{Alb}_{\text {serum }}=$ Albumin in urine and serum; $\mathrm{ACR}=$ urine albumin:creatinine ratio; $\mathrm{Cr}_{\text {urine }}, \mathrm{Cr}_{\text {serum }}=$ creatinine in urine and serum; 24-hour $\mathrm{GF}=$ glomerular filtrate formed per $24 \mathrm{~h}$; BSA = body surface area.
(A) F.E.alb relative to creatinine

$=\left(\left[\mathrm{Alb}_{\text {urine }}\right] /\left[\mathrm{Alb} \mathrm{b}_{\text {serum }}\right]\right) \div\left(\left[\mathrm{Cr}_{\text {urine }}\right] /\left[\mathrm{Cr}_{\text {serum }}\right]\right)$

$=\left(\left[\mathrm{Alb}_{\text {urine }}\right] /\left[\mathrm{Cr}_{\text {urine }}\right]\right) \times\left(\left[\mathrm{Cr}_{\text {serum }}\right] /\left[\mathrm{Alb}_{\text {serum }}\right]\right)$

$=\mathrm{ACR}\left[\mathrm{Cr}_{\text {serum }}\right] /\left[\mathrm{Alb}_{\text {serum }}\right]$

(B) F.E.alb relative to eGFR

$=$ albumin clearance $\div$ GFR

$=\left(24\right.$-hour albumin excretion $\left./\left[\mathrm{Alb}_{\text {serum }}\right]\right) \div 24$-hour GF

$=\left(24\right.$-hour albumin excretion per unit BSA $\left./\left[\mathrm{Alb}_{\text {serum }}\right]\right) \div 24$-hour GF per unit BSA

$\approx A C R /\left[\right.$ Alb $\left._{\text {serum }}\right] \div($ eGFR $\times 24 \times 60)$ \{converting eGFR to 24 -hour GF\}

$\approx A C R /\left(e G F R \times 1,440 \times\left[\mathrm{Alb}_{\text {serum }}\right]\right)$

Omitting serum albumin (see text) and the time constant gives: ACR/eGFR cally preferable to consider a single molecule and we will therefore consider albuminuria in the below discussion. However, the same arguments can be applied equally to total proteinuria.

\section{Options to Correct for GFR}

Measurement of the fractional excretion of albumin, i.e. the clearance of albumin relative to the GFR, is not a new concept. Some studies have corrected proteinuria for measured GFR, e.g. using iohexol clearance [6]. This is not, however, feasible in routine clinical practice. Measuring the fractional excretion of albumin relative to creatinine is a simpler alternative, since this does not require additional information beyond that which is routinely available to the nephrologist. This too has been performed in studies of interventions affecting both proteinuria and GFR [7], but has not been advocated for routine clinical practice. Since urinary albumin excretion is now routinely quantified by the urinary ACR, correction for reduced glomerular mass could be performed very simply by multiplying by the serum creatinine concentration (fig. 2). Formulae for fractional excretion of albumin will include serum albumin in the denominator, but the effect of this may be slight since in nephrotic hypoalbuminaemic patients management decisions will not be affected by refining the indexing with serum albumin. Consideration of both serum and urine creatinine means that variation in muscle mass is not an issue with this approach. However, using creatinine clearance as a surrogate for GFR causes problems at lower GFR due to tubular creatinine secretion and would make comparisons between individuals with low and higher GFR difficult.
The advent of routine estimated GFR (eGFR) reporting now gives the opportunity of correcting albuminuria/ proteinuria by dividing by eGFR. Original demonstration of the validity of protein:creatinine ratio (PCR) as a measure of 24-hour urine protein leak was based on the close correlation between PCR and 24-hour proteinuria per unit body surface area [8]. Since eGFR is an estimate of GFR per unit body surface area, dividing ACR by eGFR is consequently a logical procedure (fig. 2). Of note, the resulting dimensions are incorrect since this approach is based on the correlation between ACR and albumin excretion per unit body surface area. This is of course simply another expression of the fractional excretion of albumin, but by using eGFR (based on serum creatinine measurement) as denominator avoids the problems associated with creatinine clearance and tubular creatinine secretion. Correcting proteinuria or albuminuria in this way seems the most attractive method of performing a logical assessment of filtration barrier disease. Disadvantages of this approach are those inherent in formula-based estimation of GFR including underestimation of true GFR at high levels (CKD1 and -2) and overestimation in CKD5. However, the accuracy of the MDRD 4-variable formula in CKD3 where the bulk of proteinuric patients are makes it most suitable for the indexing of proteinuria for everyday clinical practice and therapeutic decision-making.

Interestingly, another approach suggested for the consideration of nephron mass is to include data on the percentage of non-sclerosed glomeruli in those patients undergoing biopsy. Bazzi et al. [9] reported improvement in the predictive power of fractional IgG excretion relative to creatinine if this was indexed for the percentage of surviving glomeruli. In using fractional excretion relative to creatinine clearance as well as the percentage of surviving glomeruli, this approach was effectively correcting for 
nephron mass twice. Potential improvement in prediction may have reflected the failure of creatinine clearance to define the degree of nephron loss in the presence of hyperfiltration. Biopsy-based assessment of functioning nephron mass is limited by the focal nature of the assessment as well as the requirement for an invasive procedure. Such an approach cannot be put forward for day-today practice and decision-making.

\section{Prognostic Effects of Proteinuria and eGFR}

Recent studies have examined the renal and vascular prognostic impact of increasing ACR at different levels of eGFR, generating hazard ratios for specific eGFR-ACR categories $[10,11]$. This could be considered a means of performing stepwise eGFR correction of ACR. In comparing the prognostic effects of increasing albuminuria within different eGFR strata, however, different increments in albuminuria per unit eGFR are being compared. Similarly, when comparing the effects of falling eGFR within an albuminuria category, the albuminuria per unit eGFR is obviously increasing concurrently with the fall in eGFR. The relative prognostic contributions of lower GFR and greater corrected albuminuria are thus difficult to separate, but both are clearly important. Indexing albuminuria for eGFR would not make absolute eGFR any less important in management decisions.

\section{Effects of Muscle Mass}

Indexing ACR for creatinine-derived eGFR is also potentially of benefit in reducing the frequently neglected effect of muscle mass on ACR assessment. Consider two patients, one muscular and the other (perhaps elderly) with low muscle mass. If they have the same absolute degree of albuminuria, say $1 \mathrm{~g} / 24 \mathrm{~h}$, then (regardless of GFR) in the steady state their respective ACRs will be quite different. Whilst the effect of muscle mass on the relationship between serum creatinine and GFR is well recognised, the effect on ACR receives much less consideration. This has implications not just for the management of renal disease but for studies assessing prognostic implications (e.g. mortality) of albuminuria thresholds that may thus be confounded by the effects of low muscle mass [12]. Current guidelines use thresholds of ACR, a measure of total albumin leak per unit creatinine generation (i.e. per unit creatinine-generating body tissue [13]) but apply these thresholds to patients regardless of their body mass. Justification for such an approach is based on reported correlations between ACR and 24-hour total albumin excretion $[2,14]$ or predictive power of ACR for identifying particular total albuminuria thresholds [15]. However, this is in contrast to the original studies correlating PCR with 24-hour proteinuria per unit body surface area (as above [8]). A correlation between ACR and albuminuria per unit body surface area is theoretically sounder on the basis that it considers creatinine-generating muscle mass (albeit with a presumably imperfect relationship between body surface area and muscle mass). The degree of reported correlation between ACR and total 24-hour albuminuria is variable [16] and will depend on the range of muscle mass in the population studied.

Indexing albuminuria for creatinine clearance solves this problem since the fall in urinary creatinine excretion accompanying reduced muscle mass is paralleled by a proportionate fall in serum creatinine. Indexing ACR for eGFR also partially solves this problem since, as discussed above, eGFR and ACR are both measures per unit body surface area. However, formula-based GFR estimation will only correct for variations in muscle mass to the extent that these variations are predicted by the variables used in the population used to derive the equation (i.e. age, sex and race from the 4-variable MDRD equation). For an individual with a muscle mass that differs significantly from that predicted by their age, sex and race, indexing ACR for eGFR will not properly correct for the effect of muscle mass. Nevertheless, eGFR indexing will still improve on the current practice of ignoring the potential effect of muscle mass. (Some guidelines recommend sex-specific ACR thresholds for microalbuminuria definition [2], though the sex-related difference in muscle mass will have the same impact at all levels of urinary albumin leak.)

\section{Stretching the Evidence}

Regarding the definition and classification of CKD, the cut-off for defining CKD1 and -2 is that of microalbuminuria (ACR $>30 \mathrm{mg} / \mathrm{g}$ ), here again without indexing for the level of eGFR. It is plausible that microalbuminuria with normal eGFR $\left(\sim 100 \mathrm{ml} / \mathrm{min} / 1.73 \mathrm{~m}^{2}\right)$, an indexed albuminuria of $0.3 \mathrm{mg} / \mathrm{g}$ per unit of eGFR, reflects a minimal per nephron leak or even the consequence of decreased tubular reabsorption of normally filtered albumin. The same level of microalbuminuria with an eGFR of $60 \mathrm{ml} / \mathrm{min} / 1.73 \mathrm{~m}^{2}$, an indexed proteinuria of $0.5 \mathrm{mg} / \mathrm{g}$ per unit eGFR almost twice as high, may have different 
pathophysiological and prognostic implications. This may also call for different management approaches. So are we right to define CKD solely by the microalbuminuria threshold without indexing for eGFR?

Also, given the logically greater biological significance of corrected proteinuria/albuminuria, the translation of evidence based on absolute proteinuria into clinical practice should be examined. Studies referenced in national guidelines have mainly measured total 24 -hour proteinuria $[1,2]$. For example, the MDRD study found a benefit of a lower blood pressure target in patients with a 24-hour protein excretion of at least $1 \mathrm{~g}$ [17]. The average measured GFR in this population was $38 \mathrm{ml} / \mathrm{min}$, giving a corresponding 24-hour urinary protein excretion rate of approximately $25 \mathrm{mg}$ per $\mathrm{ml} / \mathrm{min}$ GFR. A patient with GFR of $100 \mathrm{ml} / \mathrm{min}$ would thus require a 24 -hour protein excretion of $2.5 \mathrm{~g}$ to manifest the same degree of corrected proteinuria. Conversely, a patient with GFR $20 \mathrm{ml} / \mathrm{min}$ would only require $0.5 \mathrm{~g} / 24 \mathrm{~h}$ proteinuria.

To take a proteinuria threshold and apply it to patients without considering the GFR of the population in which the proteinuria was significant is stepping beyond the evidence. This is particularly relevant to the debate regarding optimal use of angiotensin-converting enzyme inhibitors and angiotensin II receptor blockers. Since these agents are postulated to be of particular benefit in proteinuric patients $[2,18,19]$, failing to correct proteinuria for GFR may lead to contradictory results in studies as well as inappropriate use in practice. Patients in the REIN study had an average measured GFR of approximately 50 $\mathrm{ml} / \mathrm{min}$ [18], but many other studies of the benefits of ACE inhibition in non-diabetic CKD were performed in populations with lower excretory function [19]. Of possible relevance, subanalysis of the REIN study showed that the thresholds for favourable response to ramipril were proteinuria of $>1.5 \mathrm{~g} / 24 \mathrm{~h}$ and GFR $<45 \mathrm{ml} / \mathrm{min}$ [18]. A definition of responders based on a proteinuria:GFR ratio may have minimised and possibly unified the threshold for favourable response to treatment. The UK NICE guidelines also recommend intervention thresholds of $0.5 \mathrm{~g} / 24 \mathrm{~h}$ for ACE inhibition/ARB treatment without CKD stage or GFR qualifications [2]; this is based on meta-analyses [19] and systematic reviews [20] where a mixed group of CKD patients were investigated with different levels of GFR.

Also of relevance to the debate regarding preferential use of RAAS inhibition in CKD, proteinuria in the setting of reduced functioning renal mass is often assumed to be a reflection of glomerular capillary hypertension at remnant glomeruli [21]. Reduction in glomerular capil- lary pressure by RAAS inhibition has been proposed as fundamental to a particular benefit of these agents in proteinuric CKD. The extent to which a 'remnant nephron effect' operates in human CKD is, however, unclear. If such an effect is prevalent and is indeed reflected by proteinuria, then a progressive increase in GFR-indexed proteinuria would be expected as functioning renal mass declines. Assessment of this effect and of a genuine improvement in per-nephron proteinuria following RAAS inhibition (independent of RAAS inhibition-mediated GFR reduction [6]) therefore requires GFR indexing.

Independently of the failure to correct for any measure of GFR, current guidelines based on proteinuria/albuminuria thresholds may be stretching the evidence in applying recommendations regardless of the underlying disease. Even different forms of primary glomerular disease may show a differential impact of proteinuria level on the rate of functional decline [22]. The prognostic implications of proteinuria increments in diseases such as polycystic kidney disease or interstitial nephritis may be quite different from those in primary glomerular disorders. Differences between the proteinuric tendencies of different diseases may in fact be highlighted by GFR indexing. For example, patients with amyloidosis may remain nephrotic even at commencement of dialysis, reflecting a staggering level of proteinuria per functioning nephron. The mechanism allowing some patients to remain nephrotic despite low GFR is unknown. If the intact nephron hypothesis' of Bricker [23] is considered to be operating, then profound permeability changes at the filtration barrier of remaining intact nephrons must be present.

Qualitative aspects of proteinuria may also be important for prognostic/therapeutic implications in particular diseases (a premise on which urinary proteomics is based) and this should be remembered when total proteinuria is measured [9]. Different underlying diseases similarly may affect prognostic/therapeutic implications of eGFR levels. Despite the problems with applying proteinuria thresholds across diverse patient groups, the logic of indexing proteinuria for eGFR is nevertheless applicable to all CKD patients.

\section{Conclusion}

Nephrologists have a limited number of investigations available on which to base diagnosis, prognosis and management. Making the optimal use of these investigations is important, as illustrated by the intense debate that has 
surrounded management guidelines based on eGFR thresholds. Management of patients based on proteinuria/albuminuria without any reference to the glomerular area across which the leak is occurring is illogical. Assessment of the effect of performing a simple adjustment for eGFR is needed to inform guidelines and debates on optimal treatments. Analysis of existing cohort data to define the effect of correction should be straightforward (though care must be taken in including eGFR as a denominator where the outcome measure involves functional decline). If this is found to be of no benefit, then at least current guidelines can be defended. In the meantime, we would advocate caution in applying absolute proteinuria thresholds to patient management decisions without considering the (e)GFR of the patient or the population in which the evidence was obtained.

\section{Acknowledgement}

The authors are grateful to Dr. John Haylor for helpful discussions during the preparation of the manuscript.

\section{Disclosure Statement}

Both authors declare no competing interests.

\section{References}

1 K/DOQI Clinical Practice Guidelines for Chronic Kidney Disease: Evaluation, classification, and stratification. Am J Kidney Dis 2002;39:S1-S266

2 Early identification and management of chronic kidney disease in adults in primary and secondary care (http://www.nice.org. uk/CG73).

3 Haraldsson B, Nystrom J, Deen WM: Properties of the glomerular barrier and mechanisms of proteinuria. Physiol Rev 2008;88: 451-487.

4 Comper WD, Hilliard LM, Nikolic-Paterson DJ, Russo LM: Disease-dependent mechanisms of albuminuria. Am J Physiol Renal Physiol 2008;295:F1589-F1600.

5 Russo LM, Sandoval RM, McKee M, Osicka TM, Collins AB, Brown D, Molitoris BA, Comper WD: The normal kidney filters nephrotic levels of albumin retrieved by proximal tubule cells: retrieval is disrupted in nephrotic states. Kidney Int 2007;71:504-513.

-6 Ruggenenti P, Perna A, Remuzzi G: Retarding progression of chronic renal disease: the neglected issue of residual proteinuria. Kidney Int 2003;63:2254-2261.

$\checkmark 7$ Bertolatus JA, Friedlander MA, Scheidt C, Hunsicker LG: Urinary albumin excretion after donor nephrectomy. Am J Kidney Dis 1985;5:165-169.

$\checkmark 8$ Ginsberg JM, Chang BS, Matarese RA, Garella S: Use of single voided urine samples to estimate quantitative proteinuria. N Engl J Med 1983;309:1543-1546.

$\checkmark 9$ Bazzi C, Rizza V, Raimondi S, Casellato D, Napodano P, D’Amico G: In crescentic IgA nephropathy, fractional excretion of IgG in combination with nephron loss is the best predictor of progression and responsiveness to immunosuppression. Clin J Am Soc Nephrol 2009;4:929-935.
10 Matsushita K, van der Velde M, Astor BC, Woodward M, Levey AS, de Jong PE, Coresh J, Gansevoort RT: Association of estimated glomerular filtration rate and albuminuria with all-cause and cardiovascular mortality in general population cohorts: a collaborative meta-analysis. Lancet 2010;375:20732081

11 Hemmelgarn BR, Manns BJ, Lloyd A, James MT, Klarenbach S, Quinn RR, Wiebe N, Tonelli M: Relation between kidney function, proteinuria, and adverse outcomes. JAMA 2010;303:423-429.

12 Cirillo M, Laurenzi M, Mancini M, Zanchetti A, De Santo NG: Low muscular mass and overestimation of microalbuminuria by urinary albumin/creatinine ratio. Hypertension 2006;47:56-61.

13 Heymsfield SB, Arteaga C, McManus C, Smith J, Moffitt S: Measurement of muscle mass in humans: validity of the 24-hour urinary creatinine method. Am J Clin Nutr 1983;37:478-494.

14 Chaiken RL, Khawaja R, Bard M, EckertNorton M, Banerji MA, Lebovitz HE: Utility of untimed urinary albumin measurements in assessing albuminuria in black NIDDM subjects. Diabetes Care 1997;20:709-713.

15 Jafar TH, Chaturvedi N, Hatcher J, Levey AS: Use of albumin creatinine ratio and urine albumin concentration as a screening test for albuminuria in an Indo-Asian population. Nephrol Dial Transplant 2007;22:21942200

16 Lima CS, Bottini PV, Garlipp CR, Santos AO, Costa FF, Saad ST: Accuracy of the urinary albumin to creatinine ratio as a predictor of albuminuria in adults with sickle cell disease. J Clin Pathol 2002;55:973-975.
17 Klahr S, Levey AS, Beck GJ, Caggiula AW, Hunsicker L, Kusek JW, Striker G: The effects of dietary protein restriction and bloodpressure control on the progression of chronic renal disease. Modification of diet in renal disease study group. $\mathrm{N}$ Engl J Med 1994;330:877-884.

18 Ruggenenti P, Perna A, Gherardi G, Garini G, Zoccali C, Salvadori M, Scolari F, Schena FP, Remuzzi G: Renoprotective properties of ACE-inhibition in non-diabetic nephropathies with non-nephrotic proteinuria. Lancet 1999;354:359-364.

19 Jafar TH, Schmid CH, Landa M, Giatras I, Toto R, Remuzzi G, Maschio G, Brenner BM, Kamper A, Zucchelli P, Becker G, Himmelmann A, Bannister K, Landais P, Shahinfar S, de Jong PE, de Zeeuw D, Lau J, Levey AS: Angiotensin-converting enzyme inhibitors and progression of nondiabetic renal disease. A meta-analysis of patient-level data. Ann Intern Med 2001;135:73-87.

20 Kent DM, Jafar TH, Hayward RA, Tighiouart H, Landa M, de Jong P, de Zeeuw D, Remuzzi G, Kamper AL, Levey AS: Progression risk, urinary protein excretion, and treatment effects of angiotensin-converting enzyme inhibitors in nondiabetic kidney disease. J Am Soc Nephrol 2007;18:19591965

21 Brenner BM, Lawler EV, Mackenzie HS: The hyperfiltration theory: a paradigm shift in nephrology. Kidney Int 1996;49:1774-1777.

$\checkmark 22$ Cattran DC, Reich HN, Beanlands HJ, Miller JA, Scholey JW, Troyanov S: The impact of sex in primary glomerulonephritis. Nephrol Dial Transplant 2008;23:2247-2253.

23 Bricker NS: On the meaning of the intact nephron hypothesis. Am J Med 1969;46:111. 


\section{Editorial Comment}

R.J. Glassock, Laguna Niguel, Calif.

Ellam and El Nahas are quite correct to point out the deficiencies inherent to the use of absolute urinary protein/albumin excretion (or values corrected for levels of urinary creatinine) in the diagnostic or prognostic classification of chronic kidney disease (CKD). Indeed, four variables contribute to the absolute rate of urinary protein/albumin excretion, namely (i) the concentration of the protein in plasma (specifically its concentration gradient across the glomerular capillary wall); (ii) the flux of water across the glomerular capillary wall per unit time (the glomerular filtration rate [GFR] of the individual nephrons) and the sum total of the functioning nephrons; (iii) the permeability characteristics of the individual and collective nephrons (with respect to individual protein moieties), and (iv) the tubular reabsorptive/degradative capacity of the tubules (mainly the proximal tubule) acting upon proteins appearing in Bowman's space. Variables (i), (ii) and (iii) contribute to the filtered load of protein in individual and collective nephron populations, while variable (iv) determines the fractional excretion rate (the $\%$ of the filtered load that is excreted). When the filtered load of protein is greatly elevated, tubular reabsorptive capacity can be exceeded and at that point it is mainly the filtered load that determines absolute protein exertion. GFR, as one of the determinants of filtered load, needs to be considered in evaluation of absolute protein excretion rate, as does the plasma concentration of an individual protein moiety (e.g. albumin). The latter can be best exemplified by the torrent of urinary albumin excretion that can be induced in patients with minimal change disease (a condition associated by a marked and selective increase in glomeruli permeability to albumin) by infusion of saltpoor albumin to artificially raise the plasma albumin concentration. In these circumstances the changes in GFR, glomerular permeability and tubular reabsorption of albumin induced by the albumin infusion itself are trivial, but the filtered load is not. In most proteinuric CKDs the single nephron GFR (snGFR) is not homogeneous - enhanced heterogeneity of snGFR is characteristic of CKD. Indeed, as individual nephrons are removed from the functioning population by diseaseinduced injury, less affected nephrons undergo functional hypertrophy. Thus, the whole kidney GFR (wkGFR) may not accurately reflect the actual underlying heterogeneity of the nephron population or the extent of a decline in the functioning nephron population - undermining some of the precepts embodied in indexing of proteinuria for wkGFR. In addition, indexing the urinary albumin/protein to urinary creatinine ratio (UACR) by wkGFR (or its approximation - eGFR) results in some unwanted complexities - particularly as a prognostic tool. First, it is very clear that daily creatinine generation (and thereby urinary content of creatinine) is highly correlated with prognosis (such as ESRD or allcause mortality) perhaps because low lean body mass (sarcopenia) predicts adverse outcomes [1]. Thus, UACR is a bipolar prognostic tool - an increase in absolute albumin excretion (the numerator) is associated with poor outcomes, while decreased creatinine excretion (the denominator) is also associated with poor outcomes. The ratio of albumin and creatinine in the urine reflects the competing influences of the two parameters. Indexing UACR by eGFR does not fully resolve this conundrum, as Ellam and El Nahas conjecture, and adds a further complexity since creatinine generation is part of the calculation of both UACR and eGFR. Nonetheless, the contemporary failure to appreciate the impact of the contribution of residual nephron mass to the proper evaluation of urine protein excretion as a biomarker of disease severity (and thereby its prognosis) is a worthy point, emphasized here by Ellan and El Nahas. As noted over 50 years ago by Blainey, Hardwicke, Soothill, Cameron and co-workers [2-5], the molecular characteristics of individual proteins and their fractional excretion can have value in both diagnosis and prognosis, even at equivalent total urinary protein excretion rates and differing eGFR. This phenomenon, designated urinary protein selectivity, has been re-opened as a legitimate area of inquiry of late and is a form of 'indexing' of protein excretion, but not for residual nephron mass. Finally, as emphasized by Ellam and El Nahas, it has become quite clear that the prognostic import of total urinary total protein excretion, at equivalent levels of GFR, is not equal across all of the primary and secondary proteinuric glomerular diseases (e.g. IgA nephropathy, membranous nephropathy and focal segmental glomerulosclerosis, diabetic nephropathy) [6]. Levels of total urinary protein excretion (not indexed for GFR) that have a trivial effect on projected outcome in membranous nephropathy have a profound effect on prognosis in IgA nephropathy. While introducing some caveats 
into the formulation of Ellam and El Nahas, this editorial does not negate the hypothesis that current use of urinary protein excretion thresholds based on absolute or relative protein/albumin (UACR) excretion are 'irrational'. A great deal of work still needs to be done to make these seemingly simple and readily available tools more accurate, physiologically rational, and prognostically useful when applied to individual patients with a diversity of underlying glomerular lesions and prevailing levels of GFR. The efficiency and accuracy of current models based on assessment of UACR without regard to the prevailing level of GFR (and creatinine generation) may need to be re-assessed and re-structured [7]. Hopefully, from this search we will find practical biomarker models that optimally predict clinical risk in CKD.

\section{References}

1 Lambers Heerspink HJ, Gansevoort RT, Brenner BM, Cooper ME, Parving HH, Shahinfar S, de Zeeuw D: Comparison of different measures of urinary protein excretion for prediction of renal events. J Am Soc Nephrol 2010;21:1355-1360.

-2 Blainey JD, Brewer DB, Hardwicke J, Soothill JF: The nephrotic syndrome. Diagnosis by renal biopsy and biochemical and immunological analyses related to the response to steroid therapy. Q J Med 1960;29: 235-256.

3 Soothill JF: Studies on proteinuria with special reference to immunochemically determined differential protein clearances. Guys Hosp Rep 1962;111:365-369.

4 Hardwicke J: Renal clearance of serum proteins. Clin Chim Acta 1968; 22:45.

5 Joachim GR, Cameron JS, Schwartz M, Becker EL: Selectivity of protein excretion in patients with the nephrotic syndrome. J Clin Invest 1964;43:2332-2346.

-6 Cattran DC, Reich HN, Beanlands HJ, Miller JA, Scholey JW, Troyanov S, Genes, Gender and Glomerulonephritis Group: The impact of sex in primary glomerulonephritis. Nephrol Dial Transplant 2008;23:22472253.

7 Chronic Kidney Disease Prognosis Consortium. Matsushita K, van der Velde M, Astor BC, Woodward M, Levey AS, de Jong PE, Coresh J, Gansevoort RT, et al: Association of estimated glomerular filtration rate and albuminuria with all-cause and cardiovascular mortality in general population cohorts: a collaborative meta-analysis. Lancet 2010; 375:2073-2081. 